\title{
Bleeding as a complication of fine needle lung biopsy
}

\author{
TERENCE DOYLE, MICHAEL MULLERWORTH
}

\author{
From the Department of Radiology, University of Melbourne, and the Department of Thoracic Surgery, Royal \\ Melbourne Hospital, Melbourne, Australia
}

\begin{abstract}
Two patients in whom bleeding into the pleural space was a major complication of fine needle biopsy of the lung are described. Both had a pleural effusion before biopsy.
\end{abstract}

In the 20 years since the work of Dahlgren and Nordenstrom showed $87 \%$ diagnostic accuracy and no serious complication in 365 cases, fine needle biopsy of the lung has been accepted as a minimally invasive procedure.' It is widely and correctly considered to be safe, although there is a well recognised incidence of minor complications, principally pneumothorax. We report two major complications, one fatal.

\section{Case reports}

\section{PATIENT 1}

A 32 year old woman was admitted to our hospital with a one week history of pleuritic chest pain, fever, and cough. A plain chest radiograph and a computed tomography scan (fig la) showed a $5 \mathrm{~cm}$ left lower anterior chest wall mass adjacent to and eroding the anterior end of the left fourth rib. There was also a small left pleural effusion. She had a normal clotting profile and underwent computed tomography guided biopsy with a 20G Surgimed-Rotex screw needle, an anterior approach being used. The biopsy showed syncitia of malignant cells with small nucleoli and the microglandular pattern of adenocarcinoma. Two days after the procedure she complained of left breast pain. Chest radiographs showed a progressively increasing left pleural fluid collection (fig $1 b$ ); needle aspiration of this showed only a little bloody fluid, which did not contain any neoplastic cells. The pleural opacity persisted despite intercostal catheter drainage of fluid, which was mostly blood. Because of continued bleeding that required blood transfusion and also because of pain and fever, the patient underwent a left anterior thoracotomy 12 days after biopsy. A large blood clot was evacuated from the pleural space, and a $10 \mathrm{~cm}$ diameter mass of friable tumour and blood clot was incompletely removed from behind the 4th rib. No bleeding point was found. The final histopathological diagnosis was of poorly differentiated spindle cell sarcoma. Bleeding subsequently ceased and radiotherapy was given when the wound had healed.

PATIENT 2

A 70 year old hypertensive, cigarette smoking woman was

Address for reprint requests: Professor T Doyle, Department of Radiology, University of Otago Medical School, Dunedin, New Zealand. admitted for investigation of haemoptysis, and a right lower lobe lung mass and pleural effusion were seen on the chest radiograph and computed tomogram (fig $2 a$ ). She had a normal blood clotting profile and underwent a computed tomography guided biopsy with a 20G Surgimed-Rotex screw needle, together with aspiration of pleural fluid. Examination of both fluid and biopsy material revealed squamous cell carcinoma. Two radiotherapy treatments were given. After the biopsy recurrent right sided pleural haemorrhage (fig $2 b$ ) required intercostal tube drainage, transfusion of nine units of blood, and eventually, 19 days after biopsy, thoracotomy. A clotted haemothorax was evacuated and partial decortication was required to re-expand the lung. A small $(1.5 \mathrm{~cm}$ diameter) mass of adherent blood clot was found on the pleural surface overlying the tumour. This was removed and a possible bleeding point sealed by diathermy. The friable blood clot contained aggregates of tumour cells. An attempt was made to induce pleurodesis with $1 \%$ silver nitrate solution, but pleural bleeding continued and drains were reinserted. Despite blood transfusion and bronchial artery embolisation she eventually succumbed to persisting blood and pleural fluid loss and died 46 days after the original biopsy.

\section{Discussion}

Diagnostic needle aspiration of the lung was first performed by Leyden in 1883 to obtain pneumonic organisms. This was 12 years before the discovery of $x$ rays. Worldwide experience with the technique is now considerable. Although many needle designs are available, most are 20 to $22 \mathrm{G}$ in diameter. Published reports suggest that the complication rate is more dependent on the operator's experience than on the needle used. ${ }^{2}$ Sinner has shown that the accuracy rates are fairly similar in different series; it was $90.7 \%$ in an overview of 5300 biopsies. $^{3}$ The most common complication is pneumothorax, which occurs in $20 \%{ }^{4}$ to $34 \%$ of biopsies, up to $10 \%$ requiring treatment. Pneumothorax occurs more frequently when biopsy is performed through the anterior chest wall $(22 \%)$ than via the posterior approach $(12 \%){ }^{5}$ Spread of lung tumour along the needle track after aspiration biopsy has been reported but is extremely uncommon.

About $10 \%$ of patients experience a short period of haemoptysis immediately after biopsy and minor pleural haematomas occur in $1 \% .^{6}$ We have found only one case report of death attributable to fine needle biopsy' apart from ours, though deaths after lung biopsy with large bore needles have been reported. ${ }^{89}$ There have been two reports of potentially fatal complications associated with the fine needle technique. In one ${ }^{10}$ tracheobronchial haemorrhage resulted in cardiac arrest and in the other" acute cardiac tamponade 

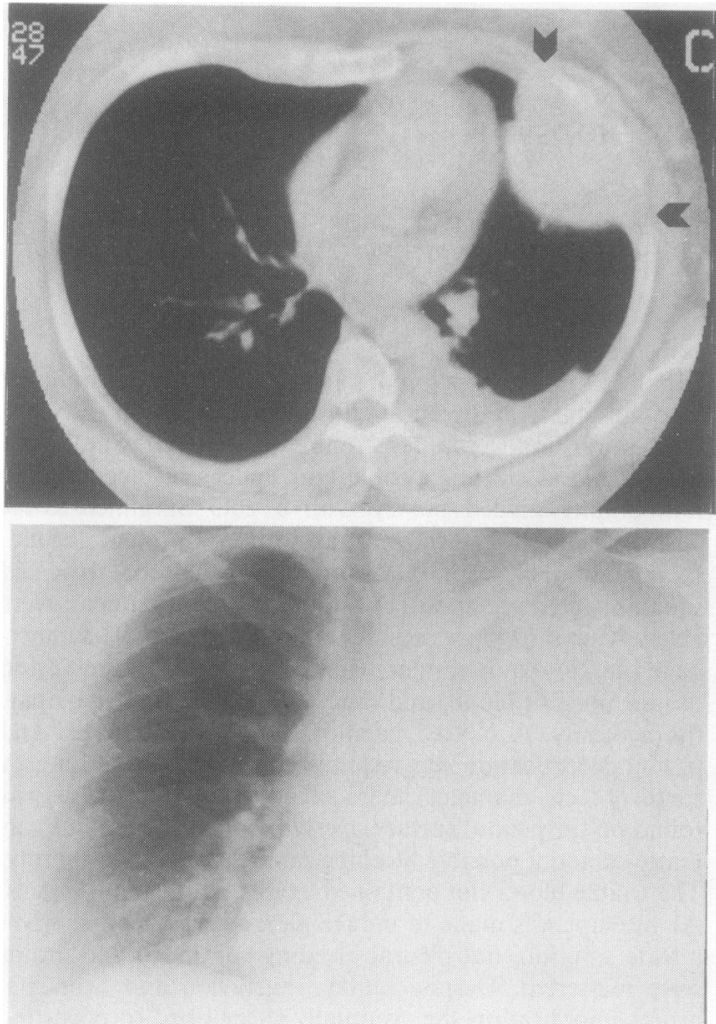

Fig 1 Patient 1: (a) Computed tomogram before biopsy showing a left anterior pleural mass infiltrating the 4th rib (arrows) (there is a left pleural effusion posteriorly); (b) erect chest film two days after biopsy, showing complete opacification of the left pleural space, which was subsequently shown to be due to blood clot.

resulted from haemorrhage of a biopsied lesion near the mediastinum, emphasising the need for care in this area.

Pleural space bleeding should be recognised as a potentially serious complication of fine needle aspiration biopsy. The problem is most likely to occur if the lesion is adjacent to a pleural surface. The combination of continuous respiratory movement and the lack of tamponade in the pleural space may lead to major haemorrhage despite a normal clotting profile, as in our cases. In the second case there was a substantial pleural effusion at the time of biopsy and this is likely also to have prevented tamponade of bleeding once the tumour had been punctured. It could reasonably be argued that pleural fluid aspiration alone would have been sufficient to make a cytological diagnosis in this case and that tumour biopsy was unnecessary or even contraindicated. At present, however, a pleural fluid collection is not generally regarded by interventional radiologists as a contraindication to biopsy of an ipsilateral lung mass. Perhaps it should be considered as a potential hazard in the future.

\section{References}

1 Nordenstrom B. A new technique for transthoracic biopsy of lung changes. Br J Radiol 1965;38:550-5.
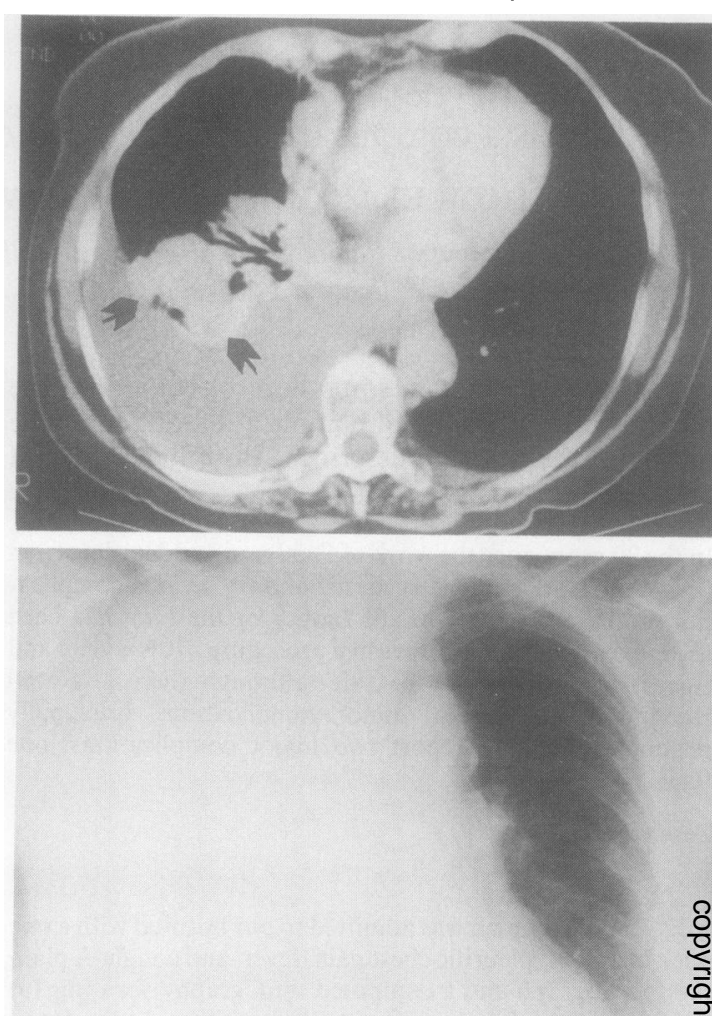

Fig 2 Patient 2: (a) Computed tomogram before biopsy showing a right lower lobe lung mass (arrows) and a right pleural fluid collection; (b) erect chest film four days after biopsy, showing the right pleural space filled with blood.

2 House AJS. Biopsy techniques in the investigation of diseases of the lung, mediastinum and chest wall. Radiol Clin North Am 1979;43:393-412.

3 Sinner WN. Pulmonary neoplasms diagnosed with transthoracic needle biopsy. Cancer 1979;43:1540-53.

4 Chin WS, Yee IST. Percutaneous aspiration biopsy of malignant lung lesions using the Chiba needle. Clin Radiol 1978;29:617-9.

5 Johnson RD, Gobien RP, Valicenti JF. Current status of $I$ radiologically directed pulmonary thin needle aspiration biopsy. Ann Clin Lab Sci 1983;13:225-39.

6 Sinner W. Complications of percutaneous transthoracic needle $N$ aspiration biopsy. Acta Radiol [Diagn](Stockh) 1976;17: 813-28.

7 Pearce JG, Patt NL. Fatal pulmonary hemorrhage after percutan- N eous aspiration lung biopsy. Am Rev Respir Dis 1974;110:346-9.

8 Roncoroni AJ, Aquiles J. Fatal complication in percutaneous biopsy of the lung [letter]. Chest 1975:68:388.

9 Milner LB, Ryan K, Bullo J. Fatal intrathoracic haemorrhage $\mathbb{D}$ after percutaneous aspiration lung biopsy. $A J R$ 1979;132: $\mathscr{A}$ 280-1.

10 Rocke DA. Percutaneous lung biopsy. Management of tracheobronchial haemorrhage. Anesthesia 1984;39:888-90.

11 Kucharczyk W, Weisbrod GL, Cooper JD, Todd T. Cardiac tamponade as a complication of thin needle aspiration lung biopsy. Chest 1982;82:120-1. 